\title{
ERRATUM
}

\section{Erratum to: Foliar $\delta^{13} \mathrm{C}$ values, photosynthetic pathway, and functional type for 238 common species on farm land and disturbed forest in Laos}

\author{
A. DE ROUW*, J.F. MAXWELL ${ }^{* *}$, and C. GIRARDIN*** \\ Institut de Recherche pour le Développement, Université Pierre et Marie Curie, 4 place Jussieu, 75252 Paris CEDEX 5 , \\ France $^{*}$ \\ Department of Biology, Chiang Mai University, Chiang Mai, Thailand** \\ Institut National de Recherche Agronomique, AgroParisTech, 78850 Thiverval Grignon, France ${ }^{* * *}$
}

\section{Erratum to: Photosynthetica \\ DOI: 10.1007/s11099-015-0081-x}

Supplementary Table 1S (published only online) was missing:

Table 1S. Foliar $\delta^{13} \mathrm{C}$, photosynthetic pathway and growth form of common species occurring in disturbed forest and fields, Houay Pano, Laos. [ $\mathrm{R}$ ] indicates species mostly regenerating in the study area by resprouting from stumps and rootstocks, [ $\mathrm{s}$ ] indicates weeds and species of disturbed environments mostly regenerating from seed.

\begin{tabular}{|c|c|c|c|}
\hline Family & Species & $\delta^{13} \mathrm{C}[\% 0]$ & Growth form, strategy \\
\hline \multicolumn{4}{|c|}{ Dicotyledonae, $C_{3}$ photosynthetic pathway } \\
\hline ACANTHACEAE & \multicolumn{2}{|l|}{ Thunbergia grandiflora (Roxb. ex Rottl.) Roxb. -29.9} & perennial vine $[\mathrm{s}$ ] \\
\hline ANACARDIACEAE & Spondias pinnata (L. f.) Kurz & -30.3 & tree $[\mathrm{R}]$ \\
\hline \multirow{2}{*}{ ANNONACEAE } & Anomianthus dulcis (Dunal) J.Sincl. & -30.8 & woody climber [ R ] \\
\hline & Cyathostemma sp 1 & -33.8 & woody climber [ R ] \\
\hline \multirow[t]{3}{*}{ APOCYNACEAE } & Amalocalyx microlobus Pierre ex Spire & -29.7 & woody climber [ R ] \\
\hline & Holarrhena pubescens Wall. ex G.Don & -29.6 & tree $[\mathrm{R}]$ \\
\hline & Ichnocarpus frutescens (L.) W.T.Aiton & -30.8 & woody climber [ R ] \\
\hline ARALIACEAE & Trevesia palmata (Roxb. ex Lindl.) Vis. & -32.0 & treelet [ R ] \\
\hline ARISTOLOCHIACEAE & Aristolochia tagala Cham. & -31.9 & perennial vine [ $\mathrm{R}$ ] \\
\hline \multirow[t]{2}{*}{ ASCLEPIADACEAE } & Zygostelma benthami Baill. & -28.9 & perennial vine $[\mathrm{R}$ ] \\
\hline & indet. liana & -32.9 & woody climber [ R ] \\
\hline \multirow[t]{5}{*}{ BIGNONIACEAE } & Fernandoa adenophylla (Wall. ex G.Don) & -29.1 & tree $[\mathrm{R}]$ \\
\hline & Steenis & & \\
\hline & Markhamia stipulata (Wall.) Seem. & -28.3 & tree $[\mathrm{R}]$ \\
\hline & Oroxylum indicum (L.) Kurz & -29.0 & tree $[\mathrm{R}]$ \\
\hline & Stereospermum neuranthum Kurz & -30.6 & $\operatorname{tree}[\mathrm{R}]$ \\
\hline BORAGINACEAE & Tournefortia montana Lour. & -33.0 & climbing shrub [ s ] \\
\hline \multirow[t]{2}{*}{ BURSERACEAE } & Canarium subulatum Guillaumin & -32.5 & tree $[\mathrm{R}]$ \\
\hline & Protium serratum (Wall. ex Colebr.) Engl. & -31.5 & tree $[\mathrm{R}]$ \\
\hline CAPPARACEAE & Stixis flavescens Pierre & -32.3 & woody climber [ R ] \\
\hline \multirow[t]{3}{*}{ CELASTRACEAE } & Celastrus paniculatus Willd. & -33.1 & woody climber [ R ] \\
\hline & Euonymus cochinchinensis Pierre & -30.8 & woody climber [ R ] \\
\hline & Reissantia indica (Willd.) N.Hallé & -30.4 & woody climber [ R ] \\
\hline \multirow[t]{4}{*}{ COMBRETACEAE } & Combretum decandrum Jacq. & -29.4 & woody climber [ R ] \\
\hline & Combretum quadrangulare Kurz & -30.2 & woody climber [ R ] \\
\hline & Combretum sp 1 & -30.6 & woody climber [ R ] \\
\hline & Quisqualis indica $\mathrm{L}$. & -27.1 & woody climber [ R ] \\
\hline \multirow[t]{9}{*}{ COMPOSITAE } & Ageratum conyzoides $\mathrm{L}$. & -31.0 & annual herb [s ] \\
\hline & Bidens pilosa $\mathrm{L}$. & -31.0 & annual herb [ s ] \\
\hline & Blumea balsamifera (L.) DC. & -32.2 & shrub or herb [ s ] \\
\hline & Blumea lacera (Burm.f.) DC. & -32.4 & annual herb $[\mathrm{s}]$ \\
\hline & Chromolaena odorata (L.) R.M.King \& H.Rob. & -29.1 & perennial scandant herb [ $\mathrm{s}$ ] \\
\hline & $\begin{array}{l}\text { Conyza sumatrensis (S.F.Blake) Pruski \& } \\
\text { G.Sancho }\end{array}$ & -31.0 & annual herb $[\mathrm{s}]$ \\
\hline & Crassocephalum crepidioides (Benth.) S.Moore & -31.6 & annual herb [ s ] \\
\hline & Spilanthes paniculata Wall. ex DC. & -35.0 & annual herb [ s ] \\
\hline & Vernonia cinerea (L.) Less. & -32.8 & annual herb [ s ] \\
\hline CONNARCEAE & Connarus semidecandrus Jack & -33.1 & woody climber [ R ] \\
\hline
\end{tabular}




\begin{tabular}{|c|c|c|c|}
\hline CONVOLVULACEAE & Lepistemon binectariferum (Wall.) Kuntze & -29.5 & perennial vine $[\mathrm{s}$ ] \\
\hline \multirow[t]{3}{*}{ CUCURBITACEAE } & Momordica charantia L. & -31.7 & annual vine $[\mathrm{s}]$ \\
\hline & Momordica subangulata Blume & -29.0 & annual vine $[\mathrm{s}$ ] \\
\hline & Mukia maderaspatana (L.) M.Roem. & -29.9 & annual vine $[\mathrm{s}]$ \\
\hline EBENACEAE & $\begin{array}{l}\text { Diospyros malabarica (Desr.) Kostel. var. } \\
\text { siamensis (Hochr.) Phengklai }\end{array}$ & -31.0 & tree $[\mathrm{R}]$ \\
\hline \multirow[t]{8}{*}{ EUPHORBIACEAE } & Antidesma acidum Retz. & -30.7 & shrub or treelet $[R$ ] \\
\hline & Antidesma sootepense Craib & -34.0 & tree or treelet $[\mathrm{R}$ ] \\
\hline & Aporosa octandra (Buch.-Ham. ex D.Don) & -30.1 & shrub or tree $[R]$ \\
\hline & Baccaurea ramiflora Lour. & -29.0 & $\operatorname{tree}[\mathrm{R}]$ \\
\hline & Breynia glauca Craib & -35.2 & shrub or treelet [ R ] \\
\hline & Flueggea virosa (Roxb. ex Willd.) Royle & -29.2 & shrub $[R]$ \\
\hline & Phyllanthus urinaria $\mathrm{L}$. & -32.1 & annual herb [ s ] \\
\hline & indet. tree & -32.8 & $\operatorname{tree}[\mathrm{R}]$ \\
\hline
\end{tabular}

Table $1 S$ continues on the next page

Table $1 S$ continued

FLACOURTIACEAE HYPERICACEAE

LAMIACEAE
LAURACEAE
LEEACEAE
LEGUMINOSAE
CAESALPINIOIDEAE

LEGUMINOSAE MIMOSOIDEAE

LEGUMINOSAE PAPILIONOIDEAE
Flacourtia indica (Burm.f.) Merr. $\quad-31.1$ Cratoxylum cochinchinense (Lour.) Blume $\quad-33.0$ Cratoxylum formosum (Jacq.) Benth. \& Hook.f. -33.3 ex Dyer ssp. parviflorum (Kurz) Gog.

Gmelina arborea Roxb. $-27.3$

Litsea glutinosa (Lour.) C.B.Rob. $\quad-30.4$ Phoebe lanceolata (Nees) Nees $\quad-31.0$ Leea indica (Burm. f.) Merr. $\quad-30.2$ Bauhinia bassacensis Gagnep. $\quad-31.5$

Bauhinia malabarica Roxb. $\quad-28.8$

Bauhinia ornata var. kerrii (Gagnep.) K.Larsen -29.6

\& S.S.Larsen

Bauhinia viridescens Desv. $\quad-30.0$

Caesalpinia decapetala (Roth) Alston $\quad-32.3$

Caesalpinia digyna Rottler $\quad-34.3$

Caesalpinia enneaphylla Roxb. $\quad-30.6$

Senna garrettiana (Craib) H.S.Irwin \& Barneby -29.1

Senna hirsuta (L.) H.S.Irwin \& Barneby $\quad-30.6$

Senna tora (L.) Roxb. $\quad-33.5$

Acacia concinna (Willd.) DC. $\quad-28.5$

Acacia megaladena Desv. $\quad-29.8$

Acacia pennata (L.) Willd. $\quad-27.5$

Albizia chinensis (Osbeck) Merr. $\quad-34.3$

Albizia odoratissima (L.f.) Benth. $\quad-32.5$

Albizia procera (Roxb.) Benth. $\quad-29.9$

Entada rheedii Spreng. $\quad-30.3$

Mimosa diplotricha Sauvalle $\quad-32.4$

Mimosa pudica L. $\quad-29.4$

Cajanus crassus (King) Maesen $\quad-27.4$

Cajanus goensis Dalzell $\quad-32.9$

Callerya atropurpurea (Wall.) Schot $\quad-28.6$

Crotalaria bracteata DC. $\quad-27.0$

Crotalaria dubia Graham $\quad-29.9$

Crotalaria filiformis var. kerrii (Craib) $\quad-29.7$

Niyomdham

Crotalaria pallida Aiton $\quad-29.5$

Crotalaria sessiliflora L. $\quad-29.0$

Crotalaria verrucosa $\mathrm{L}$. $\quad-29.9$

Dalbergia cochinchinensis Pierre $\quad-32.5$

Dalbergia foliacea Wall. $\quad-31.4$

Dalbergia rimosa Roxb. $\quad-29.8$

Dalbergia sp $1 \quad-31.3$

Desmodium gangeticum (L.) DC. $\quad-35.7$

Desmodium triquetrum (L.) DC. $\quad-31.6$

Diphyllarium mekongense Gagnep. $\quad-31.1$

Flemingia stricta Roxb. $\quad-30.5$

Indigofera tinctoria $\mathrm{L}$. $\quad-32.0$

Millettia pachycarpa Benth. $\quad-28.2$ tree $[\mathrm{R}$ ]

tree $[\mathrm{R}]$

tree $[R]$

tree $[R]$

tree $[\mathrm{R}$ ]

tree $[\mathrm{R}$ ]

perennial herb [ $\mathrm{R}$ ]

woody climber $[\mathrm{R}]$

tree [ R ]

woody climber $[\mathrm{R}$ ]

shrub [ R ]

woody climber $[\mathrm{R}$ ]

woody climber [ R ]

woody climber $[\mathrm{R}]$

tree $[\mathrm{R}$ ]

tree $[\mathrm{R}]$

annual herb [ $\mathrm{s}$ ]

shrub or climber [ R ]

woody climber [ R ]

shrub or climber $[\mathrm{s}]$

tree $[\mathrm{R}$ ]

tree $[R]$

tree $[\mathrm{R}]$

woody climber [ R ]

annual vine $[\mathrm{s}$ ]

perennial herb [ $\mathrm{s}$ ]

annual to perennial vine $[\mathrm{s}$ ]

annual vine [s]

tree [ R ]

annual herb [ $\mathrm{s}$ ]

annual herb [s ]

annual herb [ $\mathrm{s}$ ]

annual herb [ s ]

annual herb [ $\mathrm{s}$ ]

annual herb [ $\mathrm{s}$ ]

tree [R ]

tree [ R ]

woody climber [ R ]

tree [ R ]

perennial undershrub [ $\mathrm{s}$ ]

undershrub [ R ]

woody climber [ R ]

undershrub [ $\mathrm{R}$ ]

pereninial herb $[\mathrm{s}$ ]

woody climber [ R ] 


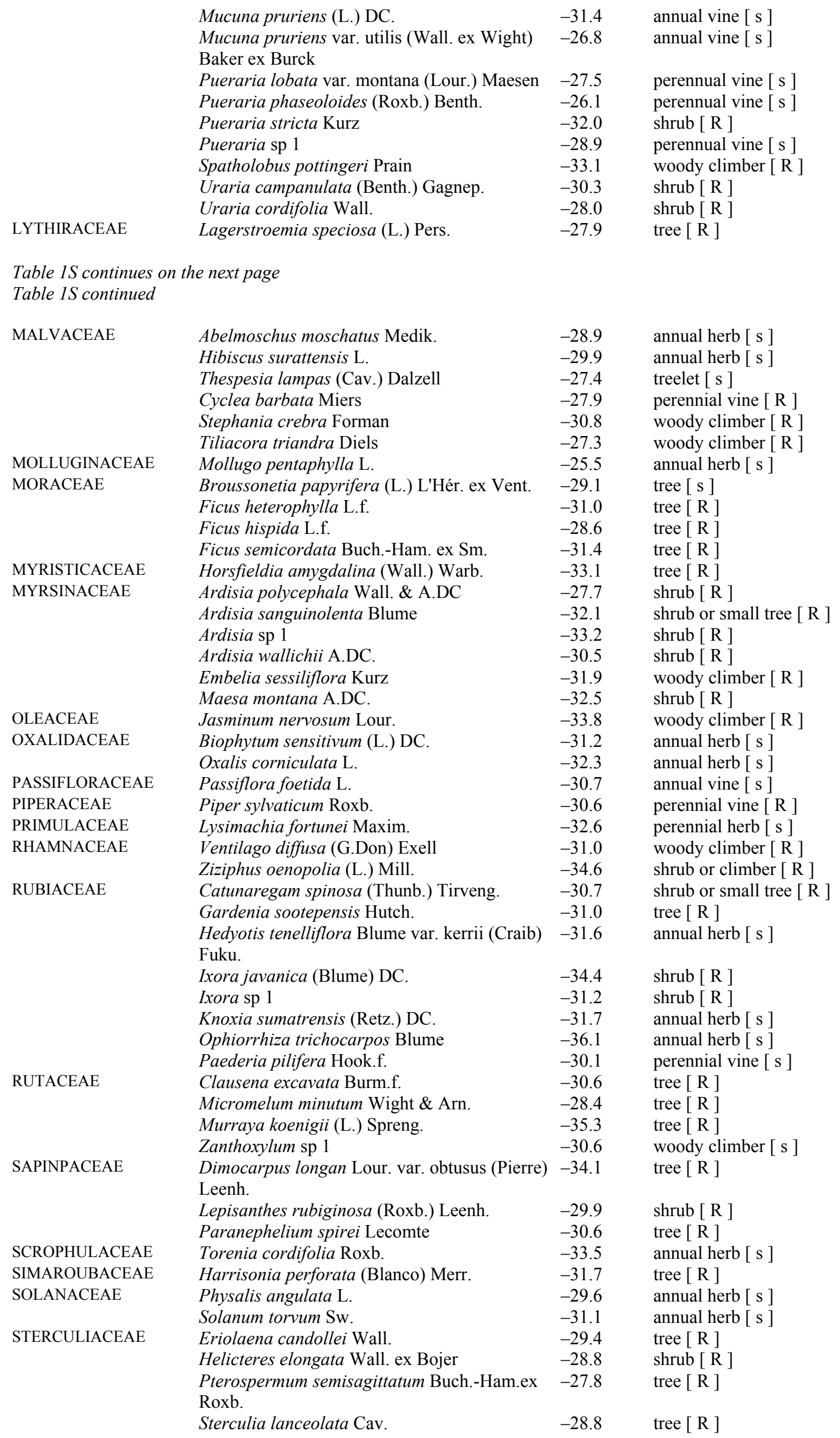




\section{SYMPLOCACEAE THYMELAECEAE} TILIACEAE

ULMACEAE
Symplocos glomerata King ex C.B. Clarke Wikstroemia meyeniana Warb.

Corchorus olitorius L.

Grewia abutilifolia Vent. ex Juss.

Grewia hirsuta Vahl

Grewia laevigata Vahl

Trema orientalis (L.) Blume
$-32.7$

$-31.6$

$-28.2$

$-29.7$

$-30.7$

$-33.4$

$-30.3$ shrub [R ]

shrub [R ]

annual herb [ $\mathrm{s}$ ]

shrub [R ]

tree $[\mathrm{R}]$

shrub [R ]

small tree $[\mathrm{s}$ ]

Table $1 S$ continues on the next page

Table $1 S$ continued

URTICACEAE
VERBENACEAE
VITACEAE

Distemon indicum Wedd.

Maoutia puya Wedd.

Cissus adnata Roxb.

\section{Dicotyledonae, $\mathrm{C}_{4}$ photosynthetic pathway}

Amaranthans hybridus L.
Amaranthus spinosus L.
Amaranthus viridis L.

Monocotyledonae, $C_{3}$ photosynthetic pathway

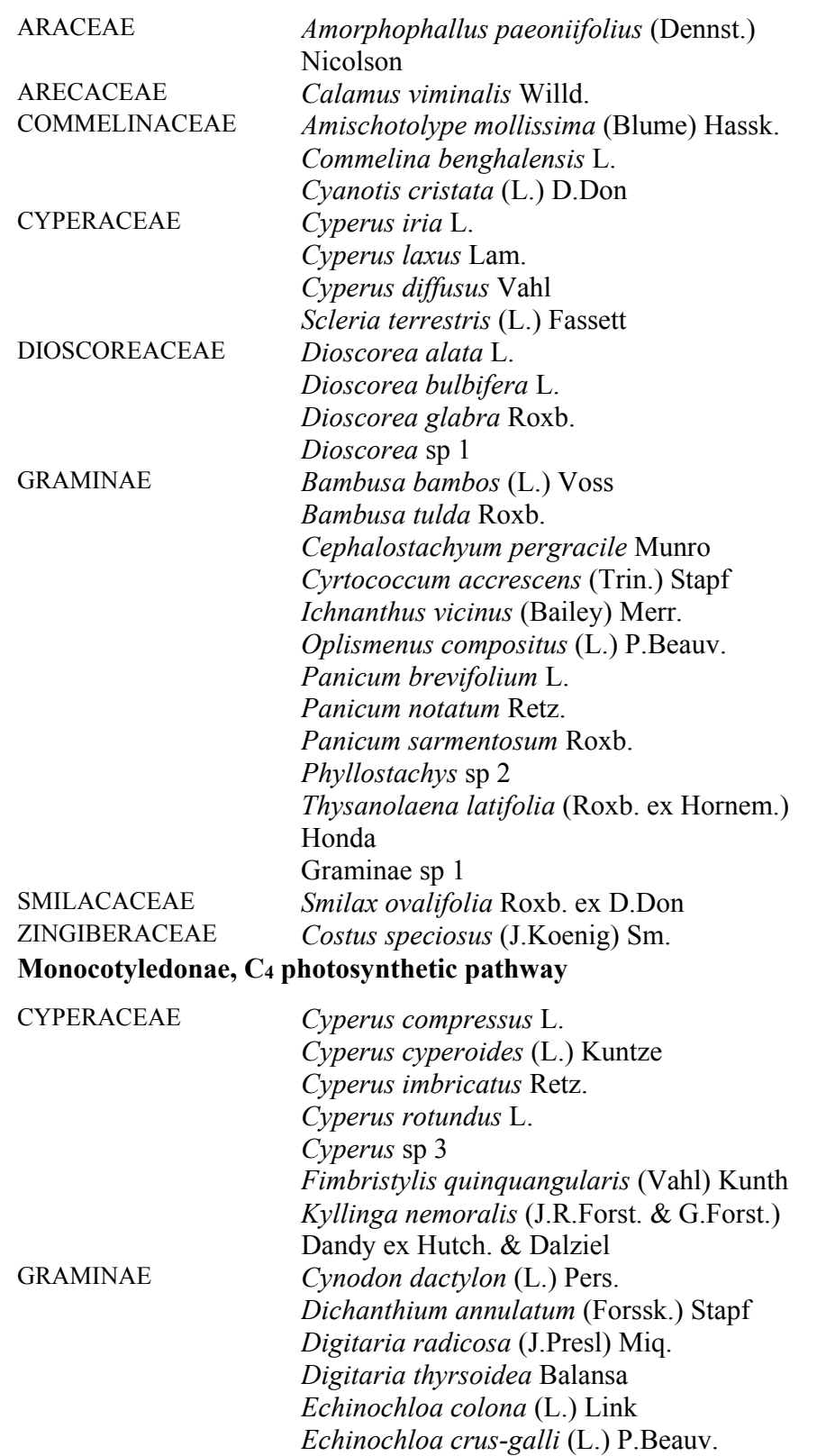

$-13.3$

$-13.8$

$-13.8$

$-$

$-29.7$

$-29.1$

$-30.1$

$-29.3$

$-29.0$

$-32.2$

$-30.1$

$-33.1$

$-29.2$

$-31.3$

$-28.3$

$-30.9$

$-32.3$

$-30.1$

$-31.4$

$-28.6$

$-31.5$

$-31.2$

$-28.9$

$-32.1$

$-31.7$

$-31.9$

$-33.0$

$-28.9$

$-29.6$

$-32.4$

$-29.3$

$-11.0$

$-10.9$

$-12.8$

$-11.8$

$-12.2$

$-11.2$

$-13.7$

$-15.2$

$-11.8$

$-11.5$

$-12.8$

$-11.0$

$-10.5$ annual herb [ s ] perennial herb [ $\mathrm{s}$ ] woody climber $[\mathrm{R}]$ perennial vine $[\mathrm{s}$ ] perennial vine $[\mathrm{s}]$

annual herb [ s ] annual herb [s ] annual herb [ $\mathrm{s}$ ]

perennial herb [ $\mathrm{s}$ ]

rattan [ R ]

robust perennial herb [ $\mathrm{R}$ ]

annual herb [s ]

perennial herb [ $\mathrm{s}$ ]

annual sedge [ $\mathrm{s}$ ]

annual sedge [ $\mathrm{s}$ ]

annual sedge [ $\mathrm{s}$ ]

climbing sedge [ $\mathrm{R}$ ]

annual vine [ $\mathrm{s}$ ]

perennial vine $[\mathrm{R}$ ]

perennial vine $[R$ ]

vine [ R ]

robust bambou [ R ]

bambou [R ]

bambou [R ]

perennial grass $[\mathrm{s}$ ]

perennial grass $[\mathrm{R}]$

perennial grass [ $\mathrm{s}$ ]

perennial grass $[\mathrm{s}$ ]

perennial grass $[\mathrm{R}$ ]

perennial grass $[\mathrm{s}]$

bambou [ R ]

perennial grass [ $\mathrm{s}$ ]

grass [ $\mathrm{s}$ ]

perennial vine $[R$ ]

perennial vine $[R]$

annual sedge [ $\mathrm{s}$ ]

perennial sedge [ $\mathrm{s}$ ]

perennial sedge $[\mathrm{s}$ ]

perennial sedge $[\mathrm{s}$ ]

sedge [ $\mathrm{s}$ ]

annual sedge [ $\mathrm{s}$ ]

perennial sedge $[\mathrm{s}$ ]

perennial grass [ $\mathrm{s}$ ]

perennial grass [ $\mathrm{s}$ ]

annual grass [ $\mathrm{s}$ ]

perennial grass [ $\mathrm{s}$ ]

annual grass [ $\mathrm{s}$ ]

annual grass [ $\mathrm{s}$ ] 
Eleusine indica (L.) Gaertn.

Imperata cylindrica (L.) Raeusch.

Microstegium ciliatum (Trin.) A.Camus

$-14.8$

$-14.0$

$-11.3$ annual grass $[\mathrm{s}]$ perennial grass $[\mathrm{s}]$ perennial grass $[\mathrm{s}]$

Table $1 S$ continues on the next page

Table IS continued

\section{Pteridophyta, $\mathrm{C}_{3}$ photosynthetic pathway}

Panicum cambogiense Balansa

Paspalum conjugatum P.J.Bergius

Paspalum urvillei Steud.

Pennisetum polystachion (L.) Schult

Saccharum spontaneum $\mathrm{L}$.

Setaria palmifolia (J.Koenig) Stapf

Urochloa panicoides P.Beauv.

\begin{tabular}{|c|c|}
\hline DENNSTAEDTIACEAE & Microlepia speluncae (L.) T.Moore \\
\hline DRYOPTERIDACEAE & $\begin{array}{l}\text { Tectaria impressa (Fée) Holttum } \\
\text { Tectaria } \mathrm{sp} 1\end{array}$ \\
\hline MARATTIACEAE & Angiopteris evecta (G.Forst.) Hoffm. \\
\hline PARKERIACEAE & Adiantum zollingeri Mett.ex Kuhn \\
\hline PTERIDACEAE & Pteris vittata $\mathrm{L}$. \\
\hline SCHIZAEACEAE & $\begin{array}{l}\text { Lygodium flexuosum (L.) Sw. } \\
\text { Lygodium polystachyum Wall. ex T. Moore }\end{array}$ \\
\hline SELAGINELLACEAE & Selaginella helferi Warb. \\
\hline THELYPTERIDACEAE & $\begin{array}{l}\text { Thelypteris ciliata (Wall. ex Benth.) Ching } \\
\text { Cyclosorus subelatus (Baker) Ching }\end{array}$ \\
\hline
\end{tabular}

\section{EUPHORBIACEAE}

LEGUMINOSAE

PAPILIONOIDEAE

Manihot esculenta Crantz

Pachyrhizus erosus (L.) Urb.

Stylosanthes guianensis (Aubl.) Sw.

Cajanus cajan (L.) Millsp.

Crotalaria juncea $\mathrm{L}$.

Monocotyledonae, cultivated, $\mathrm{C}_{3}$ photosynthetic pathway

GRAMINAE Oryza sativa L.

Monocotyledonae, cultivated, $\mathrm{C}_{4}$ photosynthetic pathway

GRAMINAE

Coix lacryma-jobi $\mathrm{L}$.

Pennisetum purpureum Schumach.

Zea mays L.
$-10.8$

$-11.7$

$-12.5$

$-12.0$

$-11.7$

$-10.3$

$-11.9$

$-28.0$

$-32.7$

$-31.4$

$-28.1$

$-31.3$

$-33.2$

$-26.9$

$-37.7$

$-31.0$

$-31.2$

$-32.2$

$-30.2$

$-30.6$

$-31.8$

$-30.2$

$-33.4$

$-31.1$

$-13.6$

$-12.2$

$-11.7$

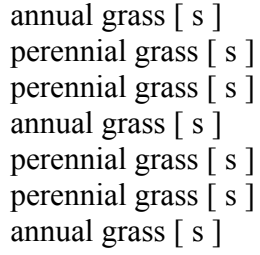

perennial fern [ R ]

perennial fern $[\mathrm{R}$ ]

fern [ R ]

robust erect fern $[\mathrm{R}$ ]

short erect fern [ $\mathrm{R}]$

perennial fern [ $\mathrm{R}$ ]

climbing perennial fern [ $\mathrm{s}$ ]

climbing perennial fern [ $\mathrm{s}]$

climbing perennial fern [ $\mathrm{s}$ ]

perennial fern [ $\mathrm{R}$ ]

perennial fern [ $R$ ]

perennial herb

annual vine

shrub

subshrub

subshrub

annual grass

annual grass

perennial grass

annual grass

The online version of the original article can be found under DOI: 10.1007/s11099-015-0081-x

${ }^{+}$Corresponding author; phone: 33(0)1 4427 72 82, fax 33(0)1 484755 34, e-mail : Anneke.De_rouw@ird.fr

The publisher and authors apologize for these errors and for inconveniences they may have caused. 